\title{
Hepatitis C Pharmacogenetics: Possible Solutions for an Existing Problem
}

\section{Sayed S Daoud ${ }^{*}$}

Department of Pharmaceutical Sciences, Center of Integrated Biotechnology, Washington State University, Pullman, Washington, USA

Over the past 20 years or so, there have been tremendous advances in our understanding of how normal cells transform to diseased/cancer cells and the importance of signaling pathways in cancer initiation and progression. This progress in scientific knowledge has resulted in the development of "personalized medicine concept" and as a proof-ofconcept, the approval of Gleevec and Herceptin. In addition, technologic advances and the use of high-throughput approaches, particularly in the last 10 years, are beginning to impact our knowledge in genome science and medical technologies. For example, advances in genotyping technologies have made it possible for the field of pharmacogenetics (the study of genetic variability in individual responses to drugs) to assume its place of importance in the medical field. Then pharmacogenetics has evolved into pharmacogenomics (the study of the roles on inherited and acquired genetic variations in drug responses) with a shift from a focus on individual candidate genes to a more comprehensive approach using Genome-Wide Association Studies (GWAS). This shift has led to the identification of genetic variations of Single Nucleotide Polymorphisms (SNPs) in the region of many genes that may play a critical role in predicting treatment response in patients at various disease states, among them patients infected with Hepatitis C Virus (HCV) [1].

An estimated $3 \%$ of the world's population-more than 170 million people-is infected with HCV. Most infections become chronic hepatitis-a condition that is incurable in many patients, leading to cirrhosis, end-stage liver disease and hepatocellular carcinoma (HCC). The current standard of care for HCV-induced chronic hepatitis is 24 or 48 weeks of therapy with pegylated interferon (PEG-IFN) and ribavirin (RBV). Response to therapy is variable, and viral as well as host characteristics can influence whether patients achieve a Sustained Virological Response (SVR) [2]. For example, patients infected with HCV genotype 1 (predominant in the US) or 4 (predominant in Africa) and who are treated for 48 weeks with PEG-IFN+RBV have less than $50 \%$ likelihood of having an SVR, whereas patients with genotype 2 (predominant in Europe) or 3 (predominant in Asia) have an SVR rate of $70 \%-80 \%$ after only 24 weeks of PEG-IFN + RBV therapy. Hence patients infected with HCV genotype 1 or 4 are poorly responsive to the antiviral therapy compared to patients infected with genotype 2 or 3. In addition, patient genetic ancestry plays a significant role in treatment outcome. For examples, African American (AA) patients with chronic HCV infection have about 50\% reduction in SVR rates with PEG+RBV treatment compared with non-Hispanic patients of European ancestry $[3,4]$. This difference is not explained by sociodemographic characteristics or compliance to treatment.

In an attempt to identify the underlying biological factors that contribute to HCV-induced HCC health disparities, we recently performed relative quantitative proteomics profiling study using eight- plex iTRAQ technique to identify differential protein expression between HCV-induced cirrhosis (CIR) andHCV-induced HCC directly compared to normal tissue samples obtained from CA patients and cross validated the identified proteins on tissue samples of AA patients [5]. The raw data were analyzed using various bioinformatics tools. Quality control analyses were accomplished using pairwise correlation plots, box plots, principal component analysis, and unsupervised hierarchical clustering. Supervised analysis was carried out to identify differentially expressed proteins. A total of 787 unique proteins were identified. Of those, about $15 \%$ were differentially expressed between normal, CIR \& HCC groups. Target validation using qRT-PCR or Western Blotting (WB), of identified proteins demonstrates racially distinct alterations in the expression of known proteins. For example, the mRNA expression levels of transferrin (TF) were 2 and 18-fold higher in CIR and HCC in AA as compared to CA. Similarly, the expression of Apolipoprotein A1 (APOA1) was 7-fold higher in HCC of AA. This increase was mirrored in the protein expression levels.

This study illustrate that proteomic identification of the molecular signatures of HCV disease progression in tissue samples using proteomic-based analyses is critically important to developing a better understanding of the biology of racial/gender disparities in HCC. This study also illustrate that additional "omics" research is needed to uncover the molecular mechanism(s) for HCC pathogenesis.

Integration of data on DNA sequences, mRNA expression, protein expression, chromosomal aberrations, chromosomal amplification and losses, single nucleotide polymorphisms (SNPs) and patterns of promoter methylation, inter alia, can all contribute to our understanding into the molecular mechanisms underlying the process of hepatocarcinogenesis as well as cancer health disparities, but each provides only partial insight. We need a research strategy that generate and integrate all of these information---and more.

\section{References}

1. Scherzer TM, Hofer H, Staettermayer AF, Rutter K, Beinhardt S, et al. (2011) Early virologic response and IL28B polymorphisms in patients with chronic hepatitis $\mathrm{C}$ genotype 3 treated with peginterferon alfa-2a and ribavirin. J Hepatol 54: 866-871

2. Afdhal NH, McHutchison JG, Zeuzem S, Mangia A, Pawlotsky JM, et al. (2011) Hepatitis C pharmacogenetics: state of the art in 2010. Hepatology 53: 336345 .

3. Howell CD, Dowling TC, Paul M, Wahed AS, Terrault NA, et al. (2008) Peginterferon pharmacokinetics in African American and Caucasian American patients with hepatitis $C$ virus genotype 1 infection. Clin Gastroenterol Hepatol 6: $575-583$.

4. Jin R, Cai L, Tan M, McHutchison JG, Dowling TC, et al. (2012) Optimum ribavirin exposure overcomes racial disparity in efficacy of peginterferon and ribavirin treatment for hepatitis C genotype 1. Am J Gastroenterol 107: 16751683.

5. Dillon ST, Bhasin MK, Feng X, Koh DK, Daoud SS (2013) Quantitative proteomic analysis in HCV-induced HCC reveals sets of proteins with potential significance for racial disparity. J Trans Med 11: 239-253.

*Corresponding author: Sayed S Daoud, Department of Pharmaceutical Sciences, Washington State University, Pullman, Washington, USA, Tel: (509) 335-8910; E-mail: daoud@wsu.edu

Received November 01, 2013; Accepted November 05, 2013; Published November 15, 2013

Citation: Daoud SS (2013) Hepatitis C Pharmacogenetics: Possible Solutions for an Existing Problem. J Pharmacogenom Pharmacoproteomics 4: e135. doi:10.4172/2153-0645.1000e135

Copyright: (c) 2013 Daoud SS. This is an open-access article distributed under the terms of the Creative Commons Attribution License, which permits unrestricted use, distribution, and reproduction in any medium, provided the original author and source are credited. 